\title{
Acolhimento em Saúde Mental: Operando Mudanças na Atenção Primária à Saúde
}

Admission in Mental Health: Working with Changes in Primary Health Care

Acogimiento en Salud Mental: Trabajo con los

Cambios en la Atención Primaria de Salud

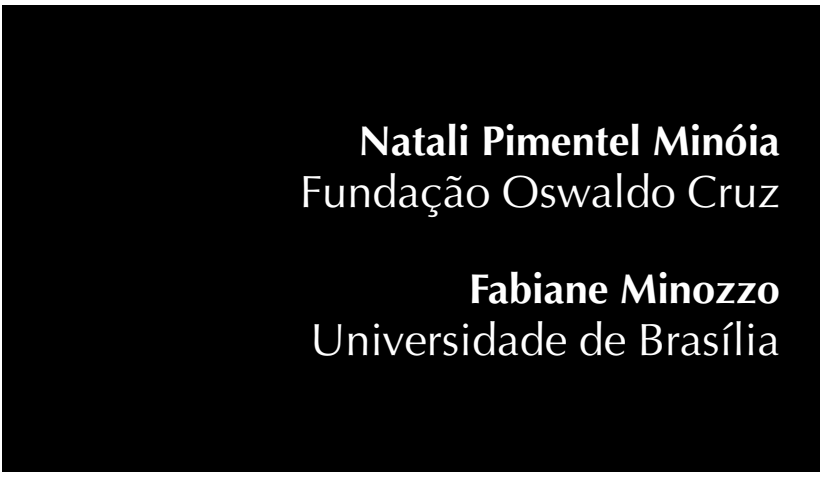

http://dx.doi.org/10.1590/1982-3703001782013

(cc) BY 
Resumo: O Movimento da Reforma Psiquiátrica evidencia a necessidade de transformação da atenção em saúde mental. A Atenção Primária à Saúde (APS) configura-se como uma estratégia de política pública que pretende promover a mudança do modelo assistencial predominante. Este artigo objetiva descrever o processo de implantação do acolhimento às pessoas em sofrimento psíquico em uma unidade de APS, no município de Porto Alegre/RS. Tem como participantes a equipe de saúde. Para tal, são apresentadas e analisadas as etapas de implantação do acolhimento, bem como os efeitos desta ação no trabalho da equipe. Discute-se o acolhimento como um operador da integralidade em saúde. Como resultados desta experiência, destaca-se a apropriação de toda a equipe de saúde pelo acolhimento dos casos de saúde mental, assim como a criação de espaços coletivos de troca entre os profissionais, para discussão dos casos complexos. Conclui-se que o acolhimento às demandas de saúde mental pelas equipes de APS auxilia na reorganização do processo de trabalho no que se refere à atenção em saúde mental, a partir de uma lógica usuário centrada, possibilita a criação de novos modos de agir e intervir da equipe e impulsiona a corresponsabilização pelas várias dimensões do adoecer e do sofrimento psíquico.

Palavras-chave: Acolhimento. Atenção Primária à Saúde. Saúde Mental.

Abstract: The psychiatric reform movement highlights the need to transform mental health care practices. Primary health care (PHC) was designed as a public policy strategy that seeks to promote changes in the predominant model of care. The objective of this article is to describe the implementation of the admission process for those who suffer from psychiatric distress in a PHC unit, in the municipality of Porto Alegre/RS. A team of healthcare providers will participate in this process. The implementation stages of the admission process are presented and analyzed as well as the effects of this action in the work carried out by the team of healthcare providers. Admission is discussed as a component of healthcare. The results of this experience highlight the need to incorporate the entire team of health care providers in the admission process and creation of a collective space designated for professional exchange amongst team members to discuss complex cases. In conclusion, the needs of the PHC teams pertaining to the mental health admission process will support the reorganization of the work pertaining to mental health care, which is centered on the patient. This will enable the creation of new methods of operation and intervention by the team, allowing for shared responsibility across the different aspects of both physical and psychological care.

Keywords: User Embracement. Primary Health Care. Mental Health.

Resumen: El Movimiento de la Reforma Psiquiátrica pone en evidencia la necesidad de transformación de la atención en salud mental. La Atención Primaria de Salud (APS) se configura como una estrategia de política pública que pretende promover el cambio del modelo asistencial predominante. Este artículo tiene el objetivo de describir el proceso de implantación del acogimiento de personas con sufrimiento psíquico en una unidad de APS en el municipio de Porto Alegre/RS. Cuenta con la participación del equipo de salud. Para ello, se presentan y analizan las etapas de implantación del acogimiento, así como los efectos de esta acción en el trabajo del equipo. Se discute el acogimiento como un operador de la integralidad en salud. Como resultados de esta experiencia se destaca la apropiación de todo el equipo de salud por el acogimiento de los casos de salud mental así como la creación de espacios colectivos de intercambio entre los profesionales, para discutir los casos complejos. Se concluye que el acogimiento de las demandas de salud mental por los equipos de APS auxilia en la reorganización del proceso de trabajo en lo que se refiere a la atención en salud mental. A partir de una lógica centrada en el usuario, hace posible la creación de nuevos modos de actuar e intervenir del equipo e impulsa la corresponsabilización por las varias dimensiones del adolecer y del sufrimiento psíquico.

Palabras clave: Acogimiento. Atención Primaria de Salud. Salud Mental. 


\section{Introdução}

O presente artigo consiste num relato de experiência que objetiva apresentar o processo de implantação do acolhimento às pessoas em sofrimento psíquico em uma unidade de Atenção Primária à Saúde (APS), no município de Porto Alegre/RS. Para tal, serão apresentadas e discutidas as etapas de implantação do acolhimento, bem como os efeitos desta ação no trabalho da equipe. Tais aspectos serão analisados a partir de tradições teóricas da saúde mental, saúde coletiva e análise institucional.

$\mathrm{O}$ acolhimento procura promover a mudança do processo de trabalho de forma a atender a todos os que procuram os serviços de saúde, buscando resolver as necessidades de saúde dos usuários. Propõe um redirecionamento das ações, tornando-as de responsabilidade de toda a equipe, promovendo a integração de saberes e práticas, ampliando sua resolubilidade.

O Movimento da Reforma Psiquiátrica evidencia a necessidade de transformação da atenção em saúde mental, buscando a substituição do modelo asilar pela atenção psicossocial. A principal diretriz do atual modelo de atenção em saúde mental consiste na ampliação e qualificação do cuidado às pessoas em sofrimento psíquico nos serviços comunitários e a reestruturação da assistência psiquiátrica hospitalar (Brasil, 2005; Costa-Rosa, 2000).

A atenção psicossocial convoca a clínica a se ampliar e a produzir outras formas de cuidado, produzindo a ampliação do objeto de trabalho e a busca de resultados eficientes, incluindo novos instrumentos. No Brasil, na perspectiva da atenção psicossocial, a rede de saúde mental é composta por diversas ações e serviços, tais como, centros de atenção psicossocial (CAPS), residenciais terapêuticos, leitos de atenção integral em saúde mental em hospital geral, unidades de acolhimento, cooperativas de trabalho e geração de renda, serviços de APS, que incluem ações das equipes de Saúde da Família, Núcleos de Apoio à Saúde da Família e consultórios na rua.
Torna-se premente a reestruturação das dinâmicas de recepção e de atendimento às pessoas em grave sofrimento psíquico na APS. O acolhimento é um possível operador desta transformação (Silveira, 2003).

A implantação do acolhimento integral em saúde, que acolhe os portadores de sofrimento psíquico, apresentado neste relato, ocorreu em uma Unidade Básica de Saúde (UBS), vinculada ao Centro de Saúde-Escola Murialdo (CSEM), Escola de Saúde Pública do Rio Grande do Sul (ESP/RS), onde são oferecidos os programas de Residência em Medicina de Família e Comunidade e de residência multiprofissional. A unidade possui responsabilidade por aproximadamente 12.000 pessoas, sendo a equipe composta por médicos, enfermeiros, técnicos de enfermagem, psicólogos, nutricionistas, assistentes sociais e cirurgião dentista, além dos residentes de medicina de família e comunidade e residentes multiprofissionais. Esta unidade não possui equipes de Saúde da Família. O acolhimento em saúde mental propõe acolher pacientes que necessitam de apoio psicossocial na APS e possibilitar, através da responsabilização compartilhada, maior resolubilidade da equipe.

\section{Implantação do acolhimento em saúde mental}

A ideia inicial de oferecer o acolhimento em saúde mental nesta unidade surgiu a partir da percepção da equipe sobre a necessidade de acolher os usuários portadores de sofrimento psíquico do território adscrito. Primeiramente, buscou-se conhecer o perfil destes sujeitos e entender quais as suas necessidades em saúde mental.

Dessa forma, foi proposto o acolhimento em saúde mental, que, na primeira fase, foi realizado por uma residente de Psicologia e um residente da medicina de família e comunidade, sob supervisão de uma psicóloga, em um dia da semana, na unidade. Os demais profissionais da equipe encaminhavam os casos que acreditavam necessitar de acolhimento psicossocial. Os usuários 
que apresentavam sofrimento psíquico eram orientados a procurar a unidade no dia do acolhimento em saúde mental. O objetivo principal consistia em conhecer, acolher e atender uma demanda de pacientes de saúde mental.

A partir de reflexões da primeira fase, chegou-se a algumas considerações em relação à tarefa de acolher: a primeira consistiu na percepção de que o acolhimento deve ser integral e a criação de um acolhimento específico em saúde mental gera uma fragmentação e cisão dos sujeitos, que são acolhidos ou por "problemas do corpo" ou por "problemas da mente". A segunda consistiu na reflexão de que a tarefa de acolher é de responsabilidade de toda equipe. $\mathrm{Na}$ forma como o acolhimento estava proposto, a atenção estava centrada na psicóloga e em um dos médicos, uma vez que ninguém mais na equipe se responsabilizava por acolher os usuários portadores de sofrimento psíquico. Ainda, identificou-se que a organização inicial proposta estava em contradição aos atributos da APS de acesso e integralidade, uma vez que o acolhimento não deve ter hora e nem dia para realizar-se e sim deve acontecer a todo o momento e durante qualquer atendimento de saúde. A primeira fase de implantação foi importante para conhecer os usuários do território e as suas necessidades. A partir destas informações, estruturaram-se as primeiras ações planejadas para atender grande parte das demandas de saúde mental na UBS, como o grupo de caminhada e o grupo de saúde mental. No que se refere ao grupo de caminhada, cabe ressaltar que inicialmente tinha como público-alvo as pessoas em sofrimento psíquico intenso, mas, com o tempo, foi aberto para todos os usuários interessados nesta ação de promoção de saúde. Este fato gerou maior socialização dos usuários portadores de sofrimento psíquico com a comunidade.

Com o decorrer do tempo, o acolhimento em saúde mental sofreu reformulações e passou para a segunda fase, em que todos os profissionais da unidade se responsabilizaram por acolher os portadores de sofrimento psíquico. Nesta fase, a equipe antes responsável pelo acolhimento em saúde mental passou a realizar apoio matricial, ou seja, a apoiar os demais profissionais na avaliação e condução clínica dos casos. Para tal, discutia os casos atendidos e realizava consultas conjuntas. O objetivo consistia em apoiar a equipe de saúde na ampliação de sua clínica, buscando aumentar seu grau de autonomia no entendimento e na condução dos casos. Também se acreditava no poder do vínculo para o alcance de melhorias no quadro clínico dos usuários e que este poderia estabelecer-se com qualquer dos profissionais da equipe, bastava que estivessem dispostos a acolher as necessidades de saúde mental dos usuários.

Qualquer abordagem assistencial de um trabalhador de saúde junto a um usuário-paciente produz-se através de um trabalho vivo em ato, em um processo de relações, [...] criando-se intersubjetivamente alguns momentos interessantes, como: momentos de falas, escutas e interpretações, no que há a produção de uma acolhida ou não das intenções que as pessoas colocam neste encontro; momentos de possíveis cumplicidades, nos quais pode haver a produção de uma responsabilização em torno do problema que vai ser enfrentado, ou mesmo de momentos de confiabilidade e esperança, nos quais se produzem relações de vínculo e aceitação (Merhy, 1999, p. 7).

Com o passar do tempo, passou-se para a terceira fase, que investiu na criação de um espaço coletivo de discussão dos casos clínicos, apostando no potencial do coletivo como promotor de troca interdisciplinar e de construção conjunta de projetos terapêuticos singulares. Buscou-se aproveitar estes encontros para discutir a atenção à saúde mental na APS (atendimentos realizados, dificuldades encontradas, rede de apoio). Procurou-se priorizar a discussão dos casos em grupo e passou-se a apostar nas trocas entre os diversos profissionais presentes nos grupos de discussão. Deste modo, o acolhimento do sofrimento psíquico colocou-se como um dispositivo de transformação do processo de trabalho da equipe, potencializando novas formas de cuidar em saúde mental. Passou-se 
a discutir as práticas em saúde mental, bem como as concepções acerca da loucura.

A estratégia utilizada foi estabelecer uma reunião semanal, com a presença da preceptoria da Psicologia, para discussão dos casos acolhidos. O horário para este encontro foi acordado em reunião de planejamento, juntamente com a exposição dos objetivos da proposta. Para potencializar a discussão coletiva e a troca entre os profissionais, procurou-se discutir os casos em grupo, apostando que cada profissional pudesse aprender com o outro. Para isso, foi necessário rever o processo de trabalho da equipe e investir no potencial de sua grupalidade.

Cuidar e gerir os processos de trabalho em saúde compõem, na verdade, uma só realidade, de tal forma que não há como mudar os modos de atender a população num serviço de saúde sem que se alterem também a organização dos processos de trabalho, a dinâmica de interação da equipe, os mecanismos de planejamento, de decisão, de avaliação e de participação. Para tanto são necessários arranjos e dispositivos que interfiram nas formas de relacionamento nos serviços e nas outras esferas do sistema, garantindo práticas de co-responsabilização, de co-gestão, de grupalização (Benevides \& Passos, 2005, pp. 392-393).

Cabe ressaltar que, no horário da reunião de discussão de casos, a unidade permanecia aberta e muitos usuários a acessavam ou estavam em atendimento, o que dificultava a presença dos profissionais médicos durante todo o tempo das reuniões. Quanto a este aspecto, salienta-se a surpresa com o fato de que a troca do horário da reunião de discussão dos casos de saúde mental gerou mobilização dos médicos que demonstraram interesse em manter um horário possível que garantisse sua presença.

Desse modo, procurou-se fazer com que cada caso levado para discussão fosse discutido com o grupo. Percebeu-se que nesses espaços pode-se esboçar um processo de interdisciplinaridade na medida em que se compartilhavam os saberes na elaboração de um projeto terapêutico singular.

Observa-se neste processo que o psicólogo no Sistema Único de Saúde desenvolve ações de campo comum de conhecimento e de núcleo específico de saber, ou seja, ações comuns e ações específicas. O núcleo demarca a identidade de uma área de saber e de prática profissional e o campo envolve um espaço de limites imprecisos em que cada disciplina e profissão buscam em outras o apoio para cumprir suas tarefas teóricas e práticas (Campos, 1999).

No relato de experiência descrito neste artigo, observa-se que o processo de trabalho do psicólogo é baseado na lógica do apoio matricial. O apoio matricial em saúde mental é uma prática em que profissionais especialistas em saúde mental oferecem suporte aos demais profissionais que atuam na APS, com o objetivo de ampliar a sua resolubilidade e produzir maior responsabilização no acompanhamento e atendimento das pessoas em sofrimento psíquico, rompendo com a lógica dos encaminhamentos indiscriminados e ampliando a sua clínica.

O apoio matricial tem duas dimensões: a) dimensão assistencial, aquela que demanda uma ação clínica direta com os usuários, como por exemplo, realização de atendimentos compartilhados e atendimentos específicos pelo psicólogo e b) dimensão técnico-pedagógico, aquela que demanda uma ação e apoio educativo com e para a equipe, como por exemplo, debate de temas teóricos e discussão de casos clínicos, tal qual o espaço coletivo de discussão dos casos elucidado na terceira fase de implantação do acolhimento em saúde mental descrito neste estudo (Campos \& Domitti, 2007).

\section{Acolhimento em ato: um caso clínico}

Para exemplificar os movimentos produzidos na equipe a partir da implantação do acolhimento integral em saúde, apresenta-se um caso clínico. Trata-se de uma senhora de 73 
anos, aqui identificada com o nome fictício de Márcia, que procura a UBS para verificar sua pressão arterial. Após verificar que sua pressão estava elevada, senta-se na sala de espera e encontra com a nutricionista da equipe. Ao perceber que a usuária precisava conversar, a nutricionista leva a senhora para uma sala. Neste momento, a paciente relata que há dois meses seu filho foi internado no hospital e, logo depois, sua nora também. Seu filho teve alta, porém sua nora faleceu. Refere preocupação com sua neta, pois esta ainda não sabia sobre o falecimento de sua mãe. A nutricionista identifica que o filho de Márcia é atendido por um profissional da equipe. O caso é levado para o espaço de discussão coletiva e o profissional que atende o filho refere que a menina é muito apegada ao pai e que vai junto a todas as consultas na unidade. Explica que o pai apresenta preocupação em melhorar sua saúde, para melhor cuidar da filha.

Neste caso, procurou-se entender as razões que levaram o pai a não falar do que havia acontecido, bem como perceber que a criança já sabia de alguma maneira da morte de sua mãe. Mas, o fato de não falar à criança, não permitia o entendimento e a elaboração emocional sobre o ocorrido e, deste modo, a criança apresentava sintomas, tais como a necessidade de estar sempre 'grudada' ao pai. Percebeu-se que estava difícil para o pai falar sobre a morte da esposa e isto o impedia de conversar com sua filha. Este só pensava em 'ficar bem' para poder cuidar da menina. Mas, a avó identificava problemas nesta relação, porém não se sentia à vontade para abordar estas questões com o filho. $\mathrm{O}$ profissional que atendia o filho também não sabia como abordar o caso, uma vez que a criança comparecia sempre às consultas juntamente com o pai.

Após a análise e discussão do caso em equipe, foi proposto solicitar à avó comparecer junto com eles na unidade, no dia da próxima consulta, para que ficasse com a menina e o profissional pudesse conversar com o pai sobre seu sofrimento pela morte de sua esposa, apontando a necessidade de abordar esta questão com sua filha. O profissional conseguiu abordar a questão com o pai e dialogou sobre a importância dele contar para a filha, para que ela pudesse elaborar o ocorrido.

Neste caso, foi importante a percepção e escuta da nutricionista sobre a preocupação da avó. A profissional levou o caso para discussão e, naquele momento, descobriu-se que a família individualmente era atendida por outros profissionais da equipe e, na reunião, compartilharam-se impressões e conjuntamente chegou-se à construção de uma estratégia de apoio à família.

\section{Discussão e algumas análises}

Durante o processo de implantação do acolhimento em saúde mental na UBS foi possível perceber algumas falas e ações que chamam a atenção por apresentarem alguns analisadores significativos que permeiam a concepção acerca da atenção à saúde mental. Os analisadores são entendidos como eventos ou acontecimentos que, por condensarem questões políticas, afetivas, conflitivas do coletivo, trazem à tona dimensões de seu cotidiano dificilmente explorado. Os analisadores tornam-se dispositivos quando conseguem produzir rupturas no instituído e brechas na homogeneidade dominante, vislumbrando saídas (Barros, 1997).

No que se refere ao caso clínico elucidado, serão apresentados os seguintes analisadores: 1) Necessidade da conduta prescritiva; 2) Dificuldade de escuta dos aspectos subjetivos; 3) Prioridade para o que é físico. Tais analisadores auxiliam no entendimento de algumas dificuldades dos profissionais que atuam na APS e indicam caminhos possíveis para a efetivação do acolhimento a saúde mental na APS.

No que se refere à necessidade da conduta prescritiva, percebeu-se que os profissionais que atuavam nesta UBS, em geral, apresentavam dificuldade em saber o que fazer com o que escutavam e, para dar uma resposta imediata, ofereciam um conselho ou faziam alguma prescrição. Demonstravam 
dificuldade de suportar a espera por uma melhora e de compreender que é importante potencializar a capacidade do usuário em pensar e refletir sobre seus problemas de saúde, trabalhando não somente com a conduta prescritiva, mas auxiliando-o a ser corresponsável pelo seu tratamento e pela sua vida. No caso abordado foi a capacidade de escuta do profissional da APS, para além das questões físicas do usuário, que possibilitou a identificação de outras necessidades de saúde. O mesmo pode-se citar da relação entre a avó e a nutricionista, em que o vínculo construído entre ambas fez com que a avó tivesse a confiança de falar sobre suas questões familiares. Caso contrário, a ação da equipe poderia ter se restringido a verificar a pressão arterial da usuária e prescrever um medicamento anti-hipertensivo.

Quanto à dificuldade de escuta dos aspectos subjetivos, percebeu-se a angústia dos profissionais diante do sofrimento alheio, apresentando a necessidade de fazer algo para que o usuário pare de chorar, demonstrando dificuldade de acolher e de escutar. Geralmente, os profissionais apresentam a necessidade de frear o sofrimento, frear o choro. Não raro, a conduta mais utilizada é a prescrição de medicamentos. O caso apresentado exigiu da equipe a ampliação da clínica, ou seja, o olhar para além dos aspectos biológicos. A questão que se colocou foi a de criar um espaço que permitisse que o sofrimento viesse a tona, para que a família pudesse falar, chorar e elaborar. Apoiar o pai para que se "autorizasse" a fazer o luto pela morte de sua esposa e dar continência para que pudesse superá-lo e cuidar de sua filha.

Em relação à prioridade para o que é físico, identificou-se que no cotidiano desta UBS, muitas vezes torna-se difícil realizar o acompanhamento familiar e longitudinal, devido ao grande número de pessoas adscritas e sob sua responsabilidade, o que é diferente em uma equipe de Saúde da Família que possui como número máximo recomendado pelo Ministério da Saúde um total de quatro mil pessoas. Desse modo, alguns profissionais atuam sem se responsabilizar efetivamente por seus usuários e suas famílias. Alguns casos são priorizados em relação a outros e, comumente, o acompanhamento das questões de saúde mental é deixado de lado, priorizando-se os aspectos orgânicos.

Percebe-se, desta forma, que a longitudinalidade e a integralidade, dois dos atributos essenciais da atenção primária à saúde são negligenciados. O atributo da longitudinalidade requer a existência do aporte regular de cuidados pela equipe de saúde e seu uso consistente ao longo do tempo. Propõe que as pessoas recebam acompanhamento durante todo o ciclo da vida: nascimento, infância, adolescência e juventude, idade adulta e no processo de envelhecimento (Starfield, 2002).

A integralidade supõe a prestação, pela equipe de saúde, de um conjunto de ações que atendam as necessidades mais comuns da população adscrita e a responsabilização pela oferta de serviços em outros pontos de atenção à saúde. Implica realizar a atenção integrando ações de promoção, prevenção, assistência e reabilitação, promovendo acesso aos diferentes níveis de atenção e ofertando respostas ao conjunto (Starfield, 2002).

A integralidade pressupõe, então, que as pessoas recebam cuidados que atendam as suas necessidades de saúde, evitando-se a fragmentação. Sendo assim, quando as questões subjetivas são ignoradas ou não identificados, quando são priorizados aspectos orgânicos para a oferta de uma ação em saúde, identifica-se que não há atenção integral, fomentando-se uma cisão entre físico e psíquico. Desta forma, identifica-se a necessidade de investir em ações que promovam a integralidade, sendo o acolhimento integral em saúde um caminho a ser percorrido na APS.

A integralidade é considerada por Mattos (2001) como um valor e deve estar presente na atitude do profissional no encontro com seus pacientes, buscando reconhecer suas demandas e necessidades de saúde. A integralidade leva em consideração o sofrimento e outros aspectos envolvidos na qualidade de vida dos sujeitos e da população, evitando a fragmentação e a redução do paciente ao sistema biológico. Além disso, uma pessoa 
vista em sua integralidade pressupõe o atendimento de toda a sua complexidade e caso for necessário o suporte de um serviço especializado, ele será acionado, mas sem perder o vínculo com o serviço de Atenção Primária à Saúde.

Para o fortalecimento da integralidade nos serviços de APS, é necessário investir na formação em serviço e a saúde mental ser entendida como uma das prioridades da APS. Há ainda a necessidade de se investir na mudança curricular que inclua o trabalho no Sistema Único de Saúde, a especificidade da APS, o cuidado psicossocial, bem como de investimentos por parte da gestão (municipal, estadual e federal) no fortalecimento da saúde mental na Saúde da Família.

\section{Considerações finais}

O acolhimento na saúde apresenta-se como uma tecnologia de cuidado eficiente para reorganizar o trabalho, a partir de uma lógica usuário centrada. Percebeu-se a importância da equipe de saúde acolher os aspectos subjetivos em todas as ações que desenvolve e que não é estratégico criar um momento ou espaço exclusivo de acolhimento em saúde mental. Esta experiência possibilitou a criação de novos modos de agir e intervir dentro da equipe, que passou refletir sobre como atender a demanda de saúde mental. Foram criados os grupos de Saúde Mental, Caminhada, Acolhimento de Pais e o Serviço de Consultoria à Escola da Comunidade, além da reorganização do fluxo de atendimento dos casos de saúde mental na UBS.

Deste modo, esta prática impulsionou um processo de corresponsabilização na equipe pelas várias dimensões do adoecer e do sofrimento psíquico. Na avaliação da proposta, a equipe percebeu que o processo de discussão dos casos, gerado a partir do acolhimento de saúde mental, deve ser extrapolado para todas as linhas de cuidado, o que permite maior compreensão e troca entre os profissionais, refletindo em uma ação integral e mais resolutiva para os usuários.

Esta prática propiciou um ambiente favorável à explicitação das dificuldades da equipe em trabalhar questões ligadas à atenção aos usuários com sofrimento psíquico. Trata-se de um processo lento e não linear, de desconstrução de crenças em relação à saúde mental e na forma de pensar o cuidado. Sendo assim, esta proposta procurou estar atenta aos movimentos de mudança, mantendo-se em contínua avaliação para chegar mais próxima das reais necessidades dos usuários que procuram os serviços de saúde, investindo no dizer de Merhy (1999, p. 7) no "fabricar cotidiano dos modelos de atenção à saúde, ao nível dos serviços, explorando suas tensões constitutivas".

Além disso, este relato de experiência pretende inspirar e auxiliar os psicólogos que atuam na APS e nos Núcleos de Apoio à Saúde da Família, em que o seu trabalho pauta-se na lógica do apoio matricial. Coloca-se como um desafio ao processo de trabalho do psicólogo no Sistema Único de Saúde a necessidade do trabalho de forma integral e interdisciplinar, tal qual se almejou neste estudo. $\mathrm{Na}$ interdisciplinaridade, os diferentes saberes, práticas, valores, modos de relacionamento se encontram para atingir objetivos comuns, exigindo dos profissionais atitudes de permeabilidade aos diferentes conhecimentos e de flexibilidade frente às diversas necessidades. É um desafio que pode ser mais facilmente enfrentado com a experimentação, ou seja, com a vivência da ação de apoiar (Brasil, 2010b). 
Brasil (2005). Ministério da Saúde. Documento apresentado à Conferência Regional de Reforma dos Serviços de Saúde Mental: 15 anos depois de Caracas. Brasília, DF: Ministério da Saúde.

Brasil (2010b). Ministério da Saúde. Oficina de qualificação do NASF. (Série C. Projetos, programas e relatórios). Brasília, DF: Ministério da Saúde.

Barros, R. D. B. (1997). Dispositivos em ação: o grupo. In A. Lancetti (Org.), Saúde, loucura, subjetividade (pp. 183-191). São Paulo, SP: Hucitec.

Benevides, R., \& Passos, E. (2005) Humanização na saúde: um novo modismo? Interface - comunicação, saúde, educação, 9(17), 389-406.

Campos, G. W. S. (1999). Equipes de referência e apoio especializado matricial: um ensaio sobre a reorganização do trabalho em saúde. Ciência \& Saúde Coletiva, 4(2), 393-403.

Campos, G. W. S.,\& Domitti, A. C. (2007). Apoio matricial e equipe de referência: uma metodologia para a gestão do trabalho interdisciplinar em saúde. Cadernos de Saúde Pública, 23(2), 399-407.

Costa-Rosa, A. (2000) O modo psicossocial: um paradigma das práticas substitutivas ao modo asilar. In P. Amarante, (Org.), Ensaios: subjetividade, saúde mental, sociedade. Rio de Janeiro, RJ: Editora Fiocruz.

Mattos, R.A. (2001). Os sentidos da integralidade: algumas reflexões acerca dos valores que merecem ser defendidos. In R. Pinheiro, \& R. A. Mattos (Org.), Os sentidos da integralidade na atenção e no cuidado à saúde (pp.39-64). Rio de Janeiro, RJ: Abrasco.

Merhy, E. E. (1999) O ato de governar as tensões constitutivas do agir em saúde como desafio permanente dealgumas estratégias gerenciais. Ciência e Saúde Coletiva, 4(2), 305-314.

Silveira, D. P. (2003). Saúde pública e saúde mental: atravessamentos em análise (Dissertação de Mestrado), Fundação Oswaldo Cruz, Rio de Janeiro, RJ.

Starfield, B. (2002). Atenção primária: equilíbrio entre necessidades de saúde, serviços e tecnologia. Brasília, DF: UNESCO. 


\section{Natali Pimentel Minóia}

Mestranda em saúde da mulher e da criança pelo Instituto Fernandes Figueira (FIOCRUZ). Psicóloga especialista em Atenção Primária à Saúde - modalidade residência multiprofissional pela Escola de Saúde Pública do Rio Grande do Sul (ESP/RS). Atua na coordenação nacional da Saúde da Mulher, Ministério da Saúde. Brasil.

E-mail: nataliminoia@hotmail.com

\section{Fabiane Minozzo}

Mestre em Psicologia Clínica e Cultura pela Universidade de Brasília (UnB). Psicóloga especialista em Saúde Mental pela mesma instituição. Especialista em Atenção Primária à Saúde - modalidade residência multiprofissional pela Escola de Saúde Pública do Rio Grande do Sul (ESP/RS).

E-mail: fabiminozzo@yahoo.com.br

\section{Endereço para envio de correspondência:}

Condomínio Morada de Deus, Rua Rota do Messias, lote 30, Setor Habitacional Jardim Botânico (Lago Sul), Brasília - DF

CEP: 71680-613

Recebido: 22/08/2013, Aprovado: 14/09/2015. 